\title{
Pediatric Epilepsy: Diagnosis and Therapy, 3rd edition
}

Pellock J M, Bourgeois B F D, Dodson E W, Nordli D R Jr, Sankar R, editors. Demos Medical Publishing, New York, 2008. Hardcover, 895 pages. ISBN-10 1933864168, ISBN-13 978-1-933864-16-7. \$206.95.

Epilepsy is a complex and common disorder that often begins in infancy and childhood. The third edition of Pediatric Epilepsy: Diagnosis and Therapy is a comprehensive reference text about epilepsy in the pediatric population. In the preface, the editors, all eminent child neurologists, state that the goal of this book continues to be assisting all professionals involved in the care of pediatric patients with seizures and epilepsy. I believe that the editors and authors have accomplished this goal. The book is an excellent resource for developing a strong understanding of current practice in the diagnosis and treatment of pediatric epilepsy.

This book differs from other child neurology texts because of its focus on pediatric epilepsy. The book is well organized into 7 main sections. The first 3 sections create the foundation for a comprehensive understanding of the diagnosis of epilepsy in infancy and childhood. The section on basic mechanisms explains the pathophysiology of seizures and epilepsy in the immature brain. This section covers topics that pediatric practitioners will find particularly useful, such as channel mutations in epilepsy. This is a rapidly evolving field, and the book helped me to understand several hereditary forms of epilepsy. Section II comprehensively reviews the classification, epidemiology, etiology, and diagnosis of epilepsies in childhood. The chapter on the first of these topics offers an interesting discussion about the history of epilepsy classification and sets out the current accepted classification of epileptic seizures and the various forms of epilepsy. This is important because the selection of drug therapies is based on their efficacy for particular seizure types and/or syndromes. The 14 chapters in Section III are dedicated to the presentation of age-related syndromes, from the newborn period to adolescence. The chapter authors are international authorities in caring for patients with these epilepsy syndromes. This section will be useful to pharmacists because it discusses specific syndrome-related treatments. Knowledge about specific types of epilepsy will also enhance the pharmacist's ability to communicate with the patient and family.

Sections IV to VI cover the general principles of therapy, specific anticonvulsant medications, ketogenic diet, and epilepsy surgery. Chapters such as "Treatment Decisions in Childhood Seizures" are key to helping the novice practitioner understand 
and contribute to successful treatment, and this type of information is often difficult to find. Anticonvulsant medications are discussed in depth, and I am sure that this information will be very useful to practising pharmacists.

The final section covers the psychosocial aspects of epilepsy. The way in which this information is presented may help the reader to gain an appreciation for some of the challenges faced by children and families living with epilepsy.

There have been many advances in the understanding and treatment of epilepsy in recent years. This book will be an essential reference for pharmacists caring for children with seizures and epilepsy. I highly recommended this book to any health care practitioner who participates in the care of infants and children with epilepsy.

Aleksandra Bjelajac Mejia, BSPharm, PharmD, RPh

Department of Pharmacy Hospital for Sick Children

Toronto, Ontario 